\title{
Correction of Flow Stress for Hot Compression of INC0718 Alloy
}

\author{
Youhong $\mathrm{Ma}^{1,2}$, Fei Zhao ${ }^{1,2^{*}}$, Jing $\mathrm{He}^{1,2}$, Jialin Wang ${ }^{1,2}$
}

1.College of Materials and Metallurgy, Guizhou University, Guiyang 550025, Guizhou, China ;

2 .The key Laboratory for Mechanical Behavior and Microstructure of Materials, Guiyang550025, Gui zhou, China

*E-mail address: mm.fzhao@gzu.edu.cn

Keywords: IN718 alloy; hot deformation behavior;flow stress; temperature increase

Abstract. The hot deformation behavior of INCO718 alloy was investigated by hot compressive tests with Gleeble -3800 thermal simulator in the temperature range from $950^{\circ} \mathrm{C}$ to $1150^{\circ} \mathrm{C}$ and strain rate range from $0.1 \mathrm{~s}^{-1}$ to $10 \mathrm{~s}^{-1}$. In consideration of the temperature, the flow stress curves were corrected by one simple and effective method. The result shows that temperature increase becomes more obvious with the increasing of strain rate and decreasing deformation temperature.

\section{Introduction}

In the process of hot compression deformation, the relationship among flow stress, deformation temperature, and strain rate reflect the dynamic response of the thermodynamic parameters in the process of thermoplastic processing. In the hot compression experiment, the material flow stress behavior was studied. Because of the plastic deformation, the partial accumulated deformation energy of the specimen converts into heat energy. It will inevitably lead to the increasing of temperature, and also can cause some errors, so the result of the stress value measured in the experiment is higher than the value measured at nominal temperature ${ }^{[1]}$. For the effect of deformation heating, the flow stress curve can not really reflect the plastic deformation of the material, as a result, the error of stress caused by temperature effect during on the compression should arouse our attention. Especially, constitutive relationship and the construction of a model of the material require the test data being accurate enough, so the error should be minimized. Many scholars at home and abroad have been made related theory and experimental research on the correction of thermal simulation test of temperature. These fully proved the necessity of temperature correction ${ }^{[2-4]}$.

In this investigation, the hot deformation behavior of the alloy INCO718 were studied systematically under the temperature range from $950^{\circ} \mathrm{C}$ to $1150^{\circ} \mathrm{C}$ and strain rate range from $0.1 \mathrm{~s}^{-1}$ to $10 \mathrm{~s}^{-1}$. So far, the most common correction method is the Newton interpolation polynomial calculation. Experimental data requires ongoing fitting and interpolation. But the amount of calculation is large, and easily brings some errors in the process of fitting and interpolation. The author used a simple and effective correction temperature method that does not need interpolation calculation. With this method, the author provided an accurate model for the numerical simulation to study INCO718 alloy hot forming process, and also provided the basic data for the experimental study.

\section{Experimental methods and materials}

In this study, INCO718 nickel-based superalloy was machined into cylindrical compression test specimen with the length of $7.5 \mathrm{~mm}$ and diameter of $14 \mathrm{~mm}$ after solid solution. The hot compression test have been carried out on Gleeble-3800 thermal simulator.The constant temperature and constant strain rate compression were performed with the preset temperature and strain rate in the automatic control system. The strain rate are $0.1 \mathrm{~s}^{-1}, 1.0 \mathrm{~s}^{-1}, 5.0 \mathrm{~s}^{-1}$ and $10.0 \mathrm{~s}^{-1}$, the deformation temperature are $950^{\circ} \mathrm{C}, 1000^{\circ} \mathrm{C}, 1050^{\circ} \mathrm{C}, 1100^{\circ} \mathrm{C}$ and $1150^{\circ} \mathrm{C}$, and the deformation quantity is 0.7 . The water quenching process of speciment was conducted immediately after compression so that the deformation microstrucion of the alloy could be kept at the end of the compression. The typical 
microstructure was observed with metallographic microscope in different deformation condition. The saturated oxalic acid solution was electrolytic solution.

\section{The experimental results and analysis}

In measuring flow stress of trial, most plastic deformnation energy can be transformed into heat. Because test time is extremely short and test process close to heat insulation, so compression test at the high strain rate makes specimen temperature rising continuely, and the flow stress curve which obtained from test is not equal to flow stress under the isothermal condition. Therefore, it is necessary to make the appropriate amendments. This paper adopted the following correction method: There was a hypothesis that the test process at the high strain rate was heat insulation process and $95 \%$ of plastic deformnation energy could be transformed into heat energy. When the specimen occured plastic deformnation, temperature rise of specimen could use the following formula $\Delta T$ to calculate ${ }^{[5-7]}$.

$\Delta \mathrm{T}=\frac{0.95 \eta \int_{0}^{\varepsilon} \sigma \mathrm{d}_{\varepsilon}}{\rho \mathrm{C}}$

Where $\Delta T$ is the temperature change, $\eta=$ adiabatic correction factor, $\int_{0}^{\varepsilon} \sigma d_{\varepsilon}=$ mechanical work, $\rho=$ specific gravity $\left(8.24 \mathrm{~g} / \mathrm{cm}^{3}\right), \quad c=$ heat capacity $\left(435 \mathrm{~J} / \mathrm{Kg}^{\circ} \mathrm{C}\right)$, and 0.95 is the percentage of the mechanical work turning into heat.

At each test temperature, $\eta$ was calculated by the following formula $: \eta=\frac{\Delta T_{\text {Actual }}}{\Delta T_{\text {Adiabacit }}}$

Where $\Delta T_{\text {Actual }}$ is the temperature rise of the actual deformation, $\Delta T_{\text {Adiabacit }}$ is the temperature rise under the condition of adiabatic .

$\eta$ is between 0 and 1 , that it is the equivalent of isothermal process under low strain rate, and it is the equivalent of adiabatic process under the high strain rate. Goetz $\mathrm{R} \mathrm{L}^{[8]}$ found that $\eta$ is a heat transfer coefficient related to temperature and strain rate.

when $\dot{\varepsilon}$ are $0.1 \mathrm{~s}^{-1}, 1 \mathrm{~s}^{-1}, 5$ and $10 \mathrm{~s}^{-1}, \eta$ are $0.46,0.85,0.92$ and 0.97 . According to the Formula (1), the actual temperature of specimen in the process of the experiment is equal to temperature rise which was calculated in the high strain rate plus the installed temperature.

Under those condition, the deformation temperature were $950^{\circ} \mathrm{C}, 1000^{\circ} \mathrm{C}, 1050^{\circ} \mathrm{C}, 1100^{\circ} \mathrm{C}$ and $1150^{\circ} \mathrm{C}$, and the strain rate were $0.1 \mathrm{~s}^{-1}, 1 \mathrm{~s}^{-1}, 5 \mathrm{~s}^{-1}, 10 \mathrm{~s}^{-1}$. The change of temperature was shown in Fig.1. It shows that the temperature increase is more and more obvious with strain rate increasing at $950^{\circ} \mathrm{C}$ deformation, and the deformation time is short at high strain rate. In the process of hot compression, the alloy produced a large amount of heat which had no time to dissipation, so the internal temperature of the alloy increased sharply. Under the condition, the temperature could rise to $177^{\circ} \mathrm{C}$ when the strain rate was $10 \mathrm{~s}^{-1}$. At the same strain rate, the trend of INCO718 alloy temperature rise caused by hot compressive deformation was getting weaker with the rising of deformation temperature. As was shown in Fig. 2. 

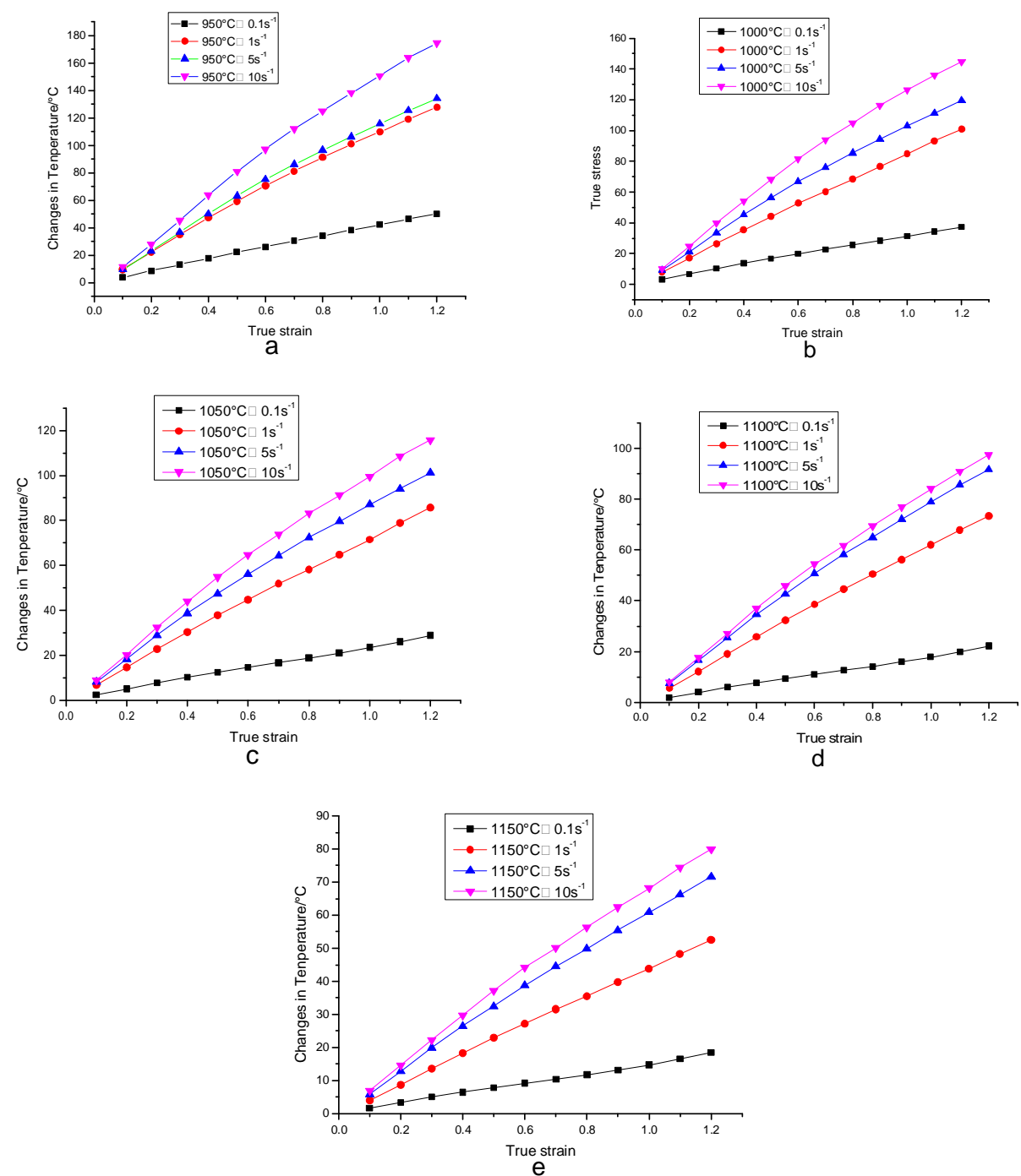

Figure1.The temperature change of the sample with different strain rate a: $950^{\circ} \mathrm{C} ; \mathrm{b}: 1000^{\circ} \mathrm{C} ; \mathrm{c}: 1050^{\circ} \mathrm{C} ; \mathrm{d}: 1100^{\circ} \mathrm{C} ; \mathrm{e}: 1150^{\circ} \mathrm{C}$

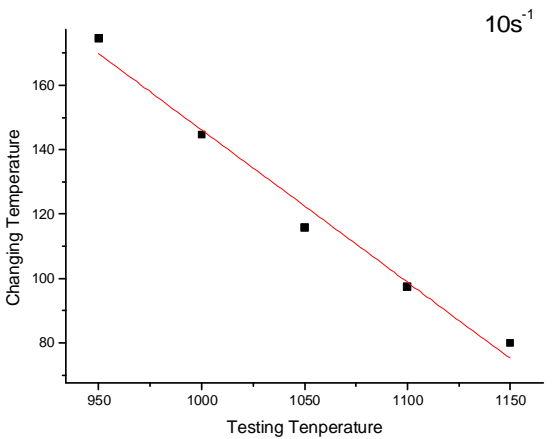

Figure2. The temperature change of the sample under different test temperature at a strain rate of $10 \mathrm{~s}^{-1}$ According to C.DEVADAS, D.BARAGAR ${ }^{[9]}$ and Gang xiao etc ${ }^{[10]}$, the alloy flow stress can use the following formula to expression with the change of temperature :

$$
\Delta \sigma=\frac{\mathrm{Q}}{\mathrm{n} \alpha \mathrm{R}}\left(\frac{1}{\mathrm{~T}}-\frac{1}{\mathrm{~T}+\Delta \mathrm{T}}\right)
$$

In this equation, $Q$ is the deformation activation energy, $R$ is molar gas constant, $n$ and $\alpha$ are material constant, $\mathrm{T}$ is the preset deformation temperature.

To solve the $n, \alpha$ and $Q$, we can use the equations below:

$\alpha=\frac{\beta}{n_{1}}$ 


$$
\begin{aligned}
& n_{1}=\left(\frac{\partial \ln \dot{\varepsilon}}{\partial \ln \sigma}\right)_{T} \\
& \beta=\left(\frac{\partial \ln \varepsilon}{\partial \sigma}\right)_{T}
\end{aligned}
$$

$H=\left[\frac{\partial \ln \varepsilon}{\partial \ln [\sinh (\alpha \sigma)]}\right]_{T}$

After calculation, $\alpha=0.00281, \beta=0.01718$ and $H=4.5459$.

In addition:

$$
Q=R\left[\frac{\partial \ln \dot{\varepsilon}}{\partial \ln [\sinh (\alpha \sigma)]}\right]_{T}\left[\frac{\partial \ln [\sinh (\beta \sigma)]}{\partial(1 / T)}\right]_{\varepsilon}
$$

$K=\partial[\ln \sinh (\alpha \sigma)] / \partial\left(\frac{1}{T}\right)$

Whence:

\section{$Q=R H K$}

According to the data of stress strain curve: $K=10623.147, Q=401.498 \mathrm{KJ} / \mathrm{mol}$. Therefore, according to the equations (3),(4),(5) and (6),(7),(8), temperature can be corrected for INCO718 alloy flow stress, and the corrected curve was shown in Fig. 3.
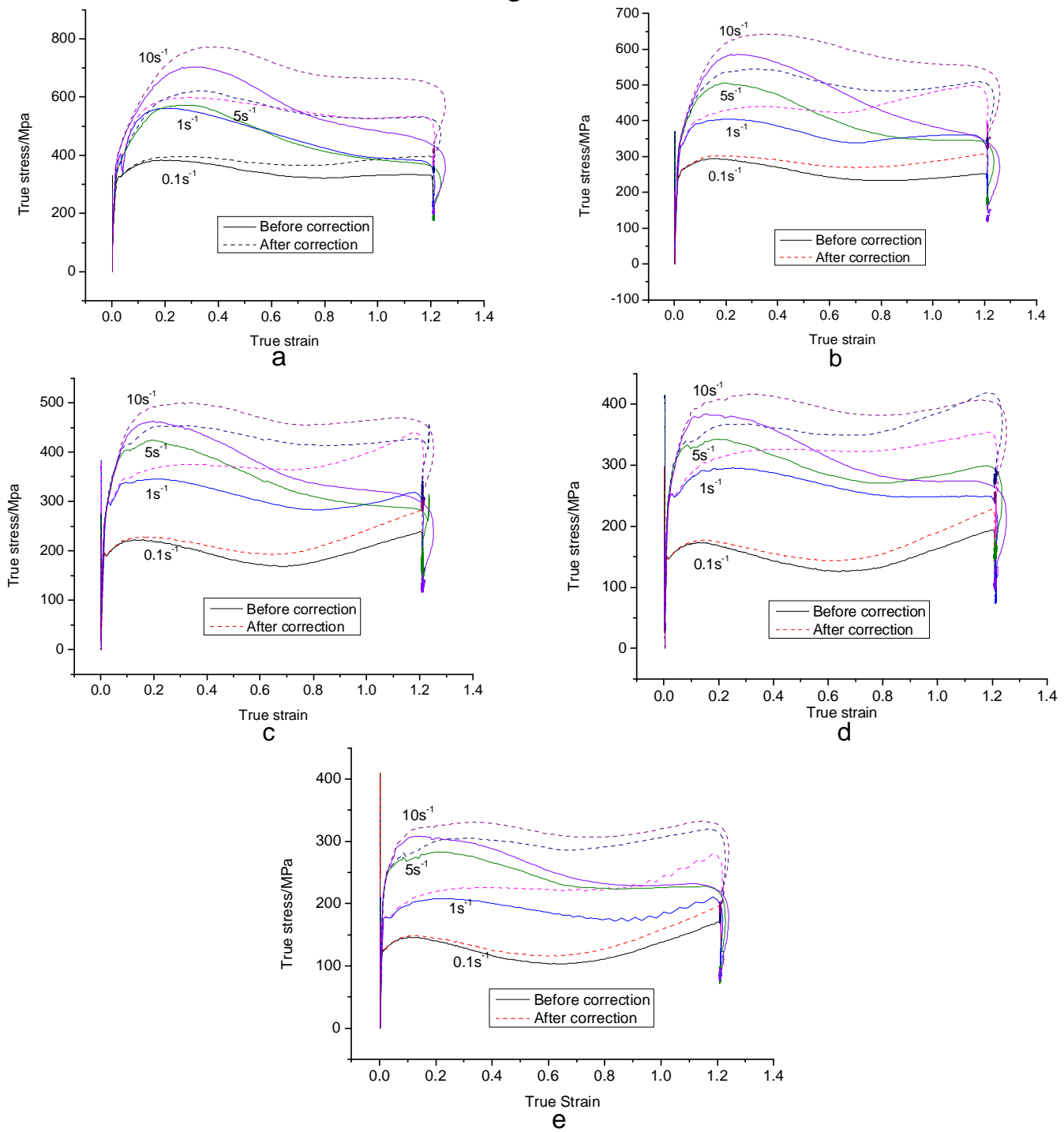

Figure 3: The comparison between temperature correction curve and not correction curve a: $950^{\circ} \mathrm{C}$ b: $1000^{\circ} \mathrm{C}$ c: $1050^{\circ} \mathrm{C}$ d: $1100^{\circ} \mathrm{C}$ e: $1150^{\circ} \mathrm{C}$ 
The comparison of flow stress curve between before and after the correction was shown in Fig.3. Accoring to the comparison between corrected true stress-strain curve and the experimental curve, the existence of temperature difference made the deformation resistance increase, particularly at low temperature. In addition, the position of the peak stress is not changed basically, so the deformation of temperature correction did not change the macro-regulation of deformation. Under low strain, the difference of two curve is very small. But after peak stress, the corrected curve becomes significantly higher than uncorrected curve. With the increasing of strain rate, the greater the deformation resistance of metals was, the bigger the true stress value was. When the strain rate was bigger, time consumption would be short at the same strain. As the deformation heat had no time to send out, thus, the temperature rise and error became larger and larger. Along with the increasing of strain rate, the stress difference caused by temperature rise also increased gradually. When the deformation temperature was $950^{\circ} \mathrm{C}$ and the strain rate was $10 \mathrm{~s}^{-1}$, the corrected stress value would be higher than the value measured in experiment 170 MPA.

A more accurate flow stress curve could be obtained after temperature amendment. As was shown in Fig.3, the true stress of the alloy increases quickly with the increasing of the strain at the beginning of the compression stage. This was due to the density of the alloy tissue dislocation increasing rapidly caused by deformation, the dislocation climbing and dislocation reaction lead to softening rate decrease relatively at the same time, and the hardening be enhanced simultaneously.After the peak of flow stress, the deformation store energy can become a driving force of recrystallization. Softening effect caused by dynamic recrystallization played a decisive role gradually in the process of deformation. When hardening rate and dynamic softening rate balanced, rheological stress reached the maximum. Along with the dynamic recrystallization, the softening rate was greater than the hardening rate, and meanwhile, the stress decreased gradually ${ }^{[10]}$.

\section{Conclusions}

(1) At constant temperature, the temperature difference is becoming more and more obvious with the increasing of strain rate. At the same strain rate, the temperature rise of INCO718 alloy decreases with the increasing of deformation temperature.

(2) IN718 alloy high temperature compression deformation true stress-strain curve has obvious dynamic recrystallization phenomenon. When it is a certain strain rate, the flow stress decreases with the increasing of deformation temperature. When it is a certain deformation temperature, the flow stress increases with the increasing of strain rate.

\section{Acknowledgment}

This research is supported by the Major Research Program (2014(6012)) and Major Fundamental Research Program ([2014]2003) of Guizhou Province, National Natural Science Foundation of China (No. 51571006).

\section{References}

[1] Xiaofei Liu , Yanshu Zhang. Quantitative analysis on hot compression test erro and software development for correction [J].FORGING \& STAMPING TECHNOLOGY, 2013, 38(1).

[2] Davenport S B, Silk N J, Sparks C N, etal. Development of constitutive equations for modelling of hot rolling[J]. Materials Science \& Technology, 2013.

[3] Weihong Zhang, Shihong Zhang.Correction of hot compress test data and constitutive equation of NiTi alloy[J].Acta metallurgica sinica, 2006, 42(10):1036-1040.

[4] Shicai Yan, Ming Cheng, Shihong Zhang, etal.High-temperature High-speed Hot Deformation Behavior of Inconel Alloy 625[J].Chinese journal of materials research, 2010, (3):239-244.

[5] Li L, Zhou J, Duszczyk J. Determination of a constitutive relationship for AZ31B magnesium alloy and validation through comparison between simulated and real extrusion[J].Journal of Materials Processing Technology, 2006, 172(3):372-380. 
[6] Charpentier P L, Stone B C, Ernst S C, etal. Characterization and modeling of the high temperature flow behavior of aluminum alloy 2024[J]. Metallurgical Transactions A, 1986, 17(12):2227-2237.

[7] Vo P, Jahazi M, Yue S, etal. Flow stress prediction during hot working of near-titanium alloys[J]. Materials Science \& Engineering A, 2007, 447(1-2):99-110.

[8] Goetz R L, Semiatin S L.The adiabatic correction factor for deformation heating during the uniaxial compression test[J].Journal of Materials Engineering \& Performance, 2001, 10(6):710-717.

[9] Devadas C, Baragar D, Ruddle G, et al. The thermal and metallurgical state of steel strip during hot rolling: Part II.Factors influencing rolling loads[J]. Metallurgical Transactions A, 1991, 22(2):321-333.

[10] Gang Xiao, Luoxing Li, Tuo Ye.Modification of flow stress curves and constitutive equations during hot plane compression deformation of 6013 aluminum alloy[J].The Chinese Journal of Nonferrous Metals, 2014:1268-1274.

[11] Lin lin.High temperature deformation behavior of GH4169 alloy[D].Harbin Institute of Technology, 2006. 